\title{
Belgeo
}

Revue belge de géographie

2| 2021

Dynamiques des campagnes et adaptations aux enjeux contemporains (Nord et Sud)

\section{Patrimoine, désenclavement et tourisme rural dans l'Anti-Atlas marocain}

Heritage, opening up and rural tourism in Moroccan Anti-Atlas

\section{André Humbert}

\section{OpenEdition}

\section{Journals}

Édition électronique

URL : https://journals.openedition.org/belgeo/49110

DOI : $10.4000 /$ belgeo.49110

ISSN : 2294-9135

Éditeur :

National Committee of Geography of Belgium, Société Royale Belge de Géographie

Référence électronique

André Humbert, " Patrimoine, désenclavement et tourisme rural dans l'Anti-Atlas marocain », Belgeo

[En ligne], 2 | 2021, mis en ligne le 11 juin 2021, consulté le 14 juin 2021. URL : http://

journals.openedition.org/belgeo/49110; DOI : https://doi.org/10.4000/belgeo.49110

Ce document a été généré automatiquement le 14 juin 2021.

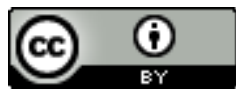

Belgeo est mis à disposition selon les termes de la licence Creative Commons Attribution 4.0 International. 


\section{Patrimoine, désenclavement et tourisme rural dans l'Anti-Atlas marocain}

Heritage, opening up and rural tourism in Moroccan Anti-Atlas

André Humbert

\section{Introduction}

1 Pour les campagnes déprimées du sud de la Méditerranée, comme celles des montagnes marocaines, le tourisme culturel - et de nature - est peut-être un levier de développement. Le tourisme n'est pas un phénomène récent au Maroc et il faut remonter bien au-delà des dernières décennies pour l'observer, sinon massivement, du moins de façon significative et ancienne. Plusieurs questions se posent néanmoins à propos des flux touristiques qui atteignent ce pays. Comment faut-il comprendre les flux globaux recensés et surtout comment interpréter les réorientations et les déséquilibres régionaux observés? Le «Sud» du pays - compris dans son acception ancienne - a évidemment bénéficié des tendances nouvelles plus orientées vers la «nature » et plus indépendantes des grands tours opérateurs. L'Anti-Atlas oriental autour des vallées du Dadès et du Draa a profité largement de l'ouverture du territoire et des liaisons électroniques, mais la partie occidentale de la même montagne, en dépit d'une richesse patrimoniale indiscutable, n'a pas connu le même succès alors qu'elle se trouve à proximité d'Agadir, un des plus grands centres touristiques du pays. C'est ce paradoxe qui va être démontré en nous appuyant sur quelques recherches mais surtout sur des observations ponctuelles, nombreuses et prolongées effectuées au cours des deux ou trois décennies passées. Ce travail de restitution d'un journal de terrain va se faire en trois temps. Il semble utile de rappeler d'abord que le Maroc est une destination touristique méditerranéenne importante et que la tradition du tourisme y est ancienne. Si cet article s'intéresse essentiellement aux problèmes du développement touristique de l'Anti-Atlas occidental, il est néanmoins intéressant 
d'évoquer aussi l'autre extrémité de la même chaîne pour souligner le déséquilibre de développement entre les deux espaces. Mais l'essentiel de cet article est consacré à l'Anti-Atlas occidental pour examiner ses forces et ses faiblesses à proximité d'un pôle majeur du tourisme mondialisé.

\section{Le Maroc : destination touristique régionale en Méditerranée}

\section{Le tourisme : un poids économique non négligeable}

2 Le Maroc n'est pas la France, ni l'Espagne pour les flux touristiques enregistrés, néanmoins ce pays affiche une bonne santé évidente - et une stabilité relative - en comparaison de certains autres pays arabes de la façade méditerranéenne. En dépit d'inflexions passagères, le pays présente une augmentation régulière des flux touristiques, atteignant environ 13 millions de visiteurs en 2019. Quelques données statistiques ${ }^{1}$ mettent en évidence la croissance régulière de cette activité au cours des dernières années et son importance dans l'économie du pays.

3 - Flux de touristes non-résidents ${ }^{2}$ :

- 2019: 13 millions environ

- $2018: 12,3$ millions

- 2017 : 11 millions

- 2015 : 10 millions dont $50 \%$ MRE

- Recettes du tourisme des non-résidents : env. 7 Md $€$ en 2018 (11 \% PIB)

- Emplois directs : 548000 (2018) soit 5 \% des emplois totaux

- Trafic-voyageurs des principaux aéroports (2019) :

- Casablanca : 10 millions

- Marrakech : 6 millions

- Agadir : 2 millions

7 On remarquera l'importance relativement forte des Marocains Résidents à l'Etranger (MRE) dans le volume total ( $50 \%$ en 2015). Ces émigrés qui «rentrent» saisonnièrement au pays, sont évidemment des touristes particuliers qui n'ont pas les mêmes modes de consommation touristique que les étrangers visiteurs, notamment pour ce qui est des modes d'hébergement.

8 La nature du tourisme a varié au cours des dernières décennies mais le Maroc, s'il a tenu à développer l'équipement de la double façade balnéaire a aussi mis à profit sa grande diversité naturelle et architecturale, de même que l'histoire déjà lointaine de son tourisme.

\section{Une tradition des « tours »}

Le Maroc a été une destination touristique bien avant que la bourgeoisie française ne colonise la médina de Marrakech (Oujamâa, 2012). L'administration du Protectorat a encouragé systématiquement des voyageurs aisés métropolitains à visiter le pays tout en régulant très largement leurs déplacements. Ainsi ont été mis en place des circuits standards, orientés vers les ressources culturelles des principales villes. Très tôt se sont développés les « tours » organisés sur le circuit dit « des villes impériales » : Marrakech, 
Fès, Meknès, Rabat (ibid., p. 290). Ces "tours" de la période coloniale reprenaient d'ailleurs une pratique ancienne de la noblesse européenne mais plus limitée dans le temps et dans l'espace. Les Français ne sont pas les seuls à s'intéresser à la richesse culturelle des villes marocaines et, pour ne citer qu'eux, dès la fin du XIX ${ }^{e}$ siècle, les Allemands publient des guides qui encouragent les voyageurs à visiter le Maroc (Scherle, 2012).

10 Evidemment, les «tours " proposés n'intéressaient pas seulement les métropolitains attirés par l'exotisme colonial. Ils étaient aussi proposés aux résidents français du Maroc. De la même façon, leur a été proposé un tourisme intérieur saisonnier et climatique. Des stations d'altitude «alpines » ont été créées sur la bordure du MoyenAtlas, à Ifrane ou à Azrou en particulier, pour accueillir la bourgeoisie fuyant la chaleur estivale dans un environnement architectural européanisé (Marzouk, 2012).

11 Mais toutes les formes touristiques au Maroc, encouragées et soutenues par les autorités coloniales, n'ont longtemps concerné que le « Maroc utile », c'est-à-dire celui de la Meseta et des bordures montagneuses qui la dominent, à l'exclusion du Sud demeuré un «Bled siba», sous la menace des tribus turbulentes, jusqu'au milieu des années 1930. Mais dès que les dernières révoltes ont été matées, dans le Sud-Est, les touristes ont été invités à découvrir l'exotisme du Sud en visitant la «Route des Kasbahs" (Oujamâa, 2012). Nous le verrons, ce mouvement précoce, a donné un avantage décisif à l'Anti-Atlas oriental sur la partie occidentale de la même chaîne de montagnes.

\section{Le déséquilibre touristique de l'Anti-Atlas}

\section{L'engouement pour le Sud-Est atlasique}

Pour des géographes (Dresch, Joly, Riser, Raynal, Troin, Weisrock), des géologues (Ball, Hecker) ou des voyageurs (Lenz, Wrage), l'Anti-Atlas est un long bourrelet de presque $700 \mathrm{~km}$ qui s'étend au sud du Haut-Atlas; sa partie orientale et le versant sud de la partie occidentale ont des caractères déjà sahariens. Très tôt, après la pacification, la partie orientale autour de Ouarzazate et au long de la vallée du Dadès a profité de l'engouement pour ce Sud «authentique » (fig. 1). A. Oujamâa (2012) signale que dès 1937, Ouarzazate est équipée de 2 hôtels, d'un gîte d'étape, de 4 café-restaurants et de divers commerces appartenant à des Européens. Dès la reprise de l'activité touristique après la Seconde Guerre mondiale, les guides attirent les touristes vers la « Route des Kasbahs » au long de la vallée du Dadès, entre Ouarzarate et Boumalne-Dadès ${ }^{3}$ (Wrage, 1967). Progressivement tout ce sud atlasique est gagné par ce tourisme à dominante culturelle, jusqu'à Errachidia et Erfoud vers l'est, et vers Zagora, au long de la vallée du Drâa. La «locomotive » de ce tourisme culturel est la «Route des Kasbahs » (Popp, 2008). Elle est reprise systématiquement dans les guides, notamment le guide Michelin de 1978 (fig. 2). 
Figuere 1. Guide touristique de Ouarzazate, en 1938.

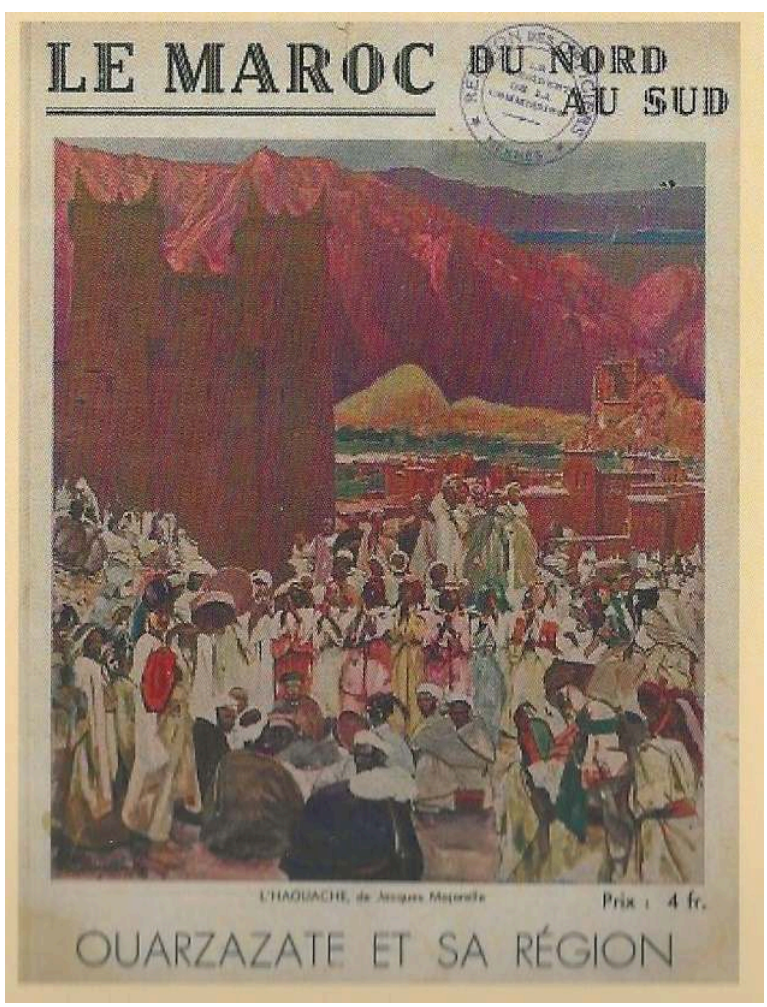

Très tôt des guides sont publiés avec la collaboration de photographes et même d'artistes réputés comme Jacques Majorelle (Oujamâa, 2012, p. 301).

Figure 2. La route des kasbahs (province de Ouarzazate).

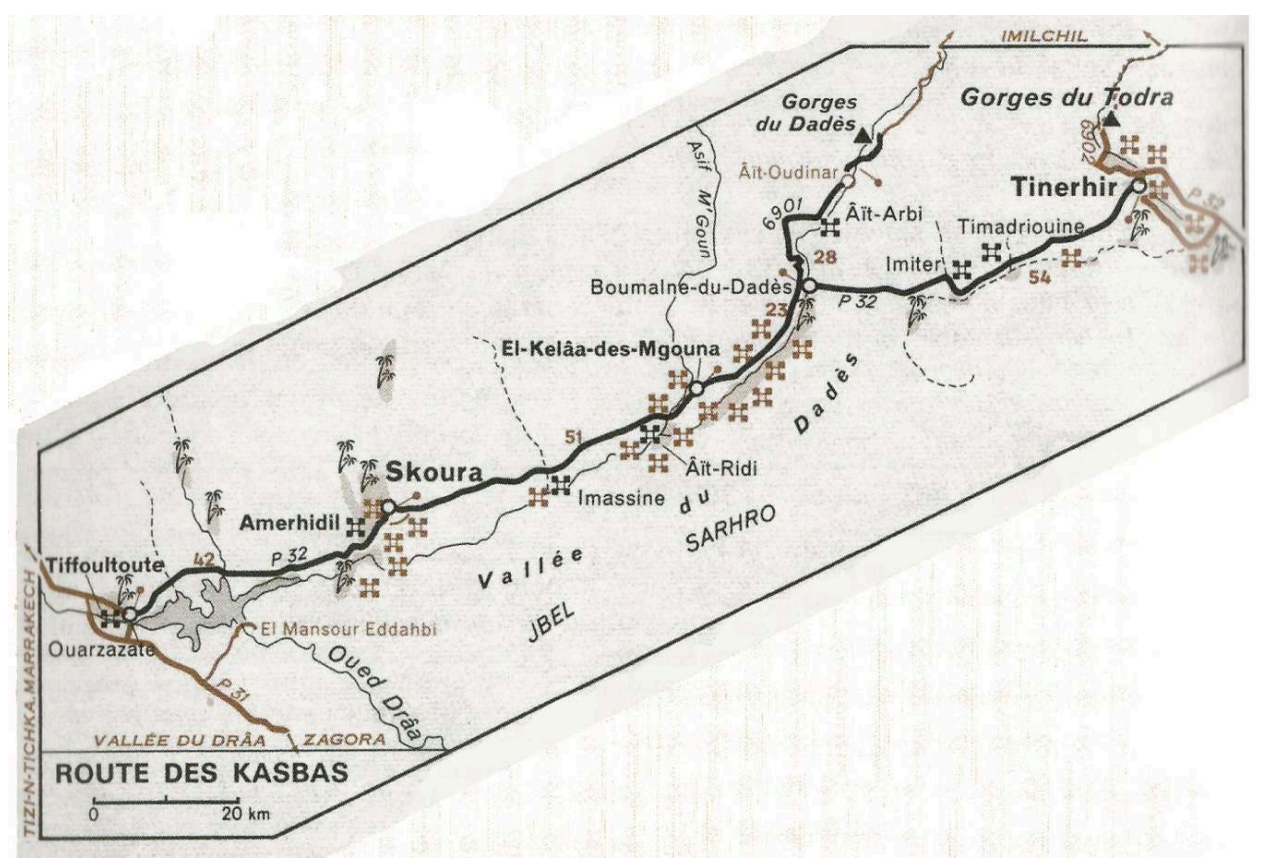

Cette route jalonnée de grandes demeures aristocratiques a, très tôt, attiré les touristes en quête d'exotisme culturel et d'images oasiennes (Oujamâa, 2012, p. 302). 
13 cette région auprès des touristes étrangers. C'est spécialement la découverte de sites naturels qui attire vers les splendides gorges du Dadès ou du Todra ou, pour les plus sportifs, la pratique du trekking à travers le massif du jbel Saghro (Leesmeister, Scherle, 2008). Plus à l'est encore, le massif dunaire de Merzouga (Erg Chebbi) a subi une avalanche calamiteuse de touristes depuis le début des années 2000 (Bouaouinane, Beckedorf \& Popp. , 2008 ; Biernest, 1998 ; Gagnol, Landel, 2016).

Les tours opérateurs se sont emparés de cette aubaine et prennent en charge les clients depuis Marrakech en leur offrant des «aventures » de quelques jours dans le Sud. Ces « excursions » étant impossibles à concevoir depuis Marrakech, distante de $200 \mathrm{~km}$ de Ouarzazate, et à quatre heures de route, un circuit de quatre ou cinq jours au moins est donc pratiquement obligatoire, pour justifier un éloignement à une distance aussi grande, avec franchissement du Haut-Atlas, de surcroît. La puissance de ces opérateurs est tellement grande qu'ils ont réussi à stériliser l'aéroport de Ouarzazate qui pourrait accueillir beaucoup plus de vols qu'il ne le fait. Une enquête menée auprès des responsables de l'aéroport et des contrôleurs du trafic permet de souligner cette indigence par rapport aux possibilités techniques des installations. Certes, avec 136000 passagers enregistrés en 2019 par rapport aux 86000 de 2018, l'aéroport est sur une pente ascendante, comme le trafic d'ensemble des aéroports marocains ; néanmoins, il ne représente que $0,54 \%$ du trafic total national ${ }^{4}$. Cependant, un certain nombre d'initiatives individuelles, favorisées par Internet, réussissent à attirer directement les amateurs de ce Sud, dans des hébergements souvent installés dans des kasbahs authentiques rénovées ou dans des simili-kasbahs hôtelières. Des travaux récents (Oussoulous, 2019) montrent d'ailleurs que les hébergements touristiques se sont multipliés dans ce Sud-Est à l'initiative de résidents étrangers. Ainsi, dans le Jbel Sarhro, le village de N'kob s'est spécialisé dans la location, par internet, de ses nombreuses kasbahs rénovées ou imitées. Plus au nord, sur la "Routes des kasbahs ", l'oasis de Skoura offre de nombreuses kasbahs encore exploitables pour le tourisme (Popp, El Fasskaoui, 2017).

Cette région du Dadès et du Drâa, prolongée jusqu'au Tafilalet, à l'est, bénéficie indiscutablement d'une antériorité touristique qui lui a été très profitable. Mais les déplacements touristiques ont été aussi facilités par un réseau de voies de circulation mises en place dès l'époque du Protectorat, ces routes empruntant les couloirs naturels que sont les grandes vallées, ou utilisant l'aération du relief, particulièrement pénétrable. Depuis longtemps, des guides et des ouvrages (Wrage, 1967) sont publiés sur toute cette région et plus récemment des universitaires en ont fait la promotion à l'aide de cartes touristiques (Ait Hamza, Popp, 2004) et d'un ouvrage sur les kasbahs (Popp, El Fasskaoui, 2017)

\section{Le décollage touristique difficile de l'Anti-Atlas occidental}

Même si l'on ne dispose pas de statistiques sûres et détaillées (tableau 1), il apparaît clairement que, compte tenu de l'indigence des équipements à l'ouest de l'énorme édifice volcanique du jbel Siroua, l'Anti-Atlas occidental, pourtant très proche d'Agadir, n'a connu jusqu'à maintenant qu'un médiocre et ponctuel développement touristique (fig. 3). 
Tableau 1. Capacités d'hébergement dans les différents secteurs de l'Anti-Atlas occidental.

\begin{tabular}{|c|c|c|c|c|c|c|}
\hline \multirow[t]{2}{*}{ Catégorie } & \multicolumn{2}{|c|}{ Tafraout/Ammeln } & \multicolumn{2}{|c|}{ Oasis présahariennes } & \multicolumn{2}{|c|}{ Reste de la montagne } \\
\hline & $\mathrm{Nb}$ établ. & Capacité & $\mathrm{Nb}$. établ. & Capacité & Nb. établ. & Capacité \\
\hline Hôtel étoilé & 4 & 309 & & & 1 & 28 \\
\hline Hôtel non classé & 10 & 306 & 1 & 20 & 2 & 54 \\
\hline Hôtel + camping & 1 & $26+14(*)$ & 2 & $42+95(*)$ & & \\
\hline Maison d'hôtes & 5 & 89 & 1 & 75 & 2 & 27 \\
\hline Gîte d'étape & 3 & 45 & 2 & 55 & 6 & 100 \\
\hline Camping & 5 & $152(*)$ & 2 & $53(*)$ & & \\
\hline Totaux & 28 & $775+166(*)$ & 10 & $192+148(*)$ & 11 & 209 \\
\hline
\end{tabular}

(*) Emplacements de tentes

D’après H. Popp et al., Carte touristique de l'Anti-Atlas occidental, 2012.

Figure 3. L'Anti-Atlas occidental : quelques sites et phénomènes touristiques cités.

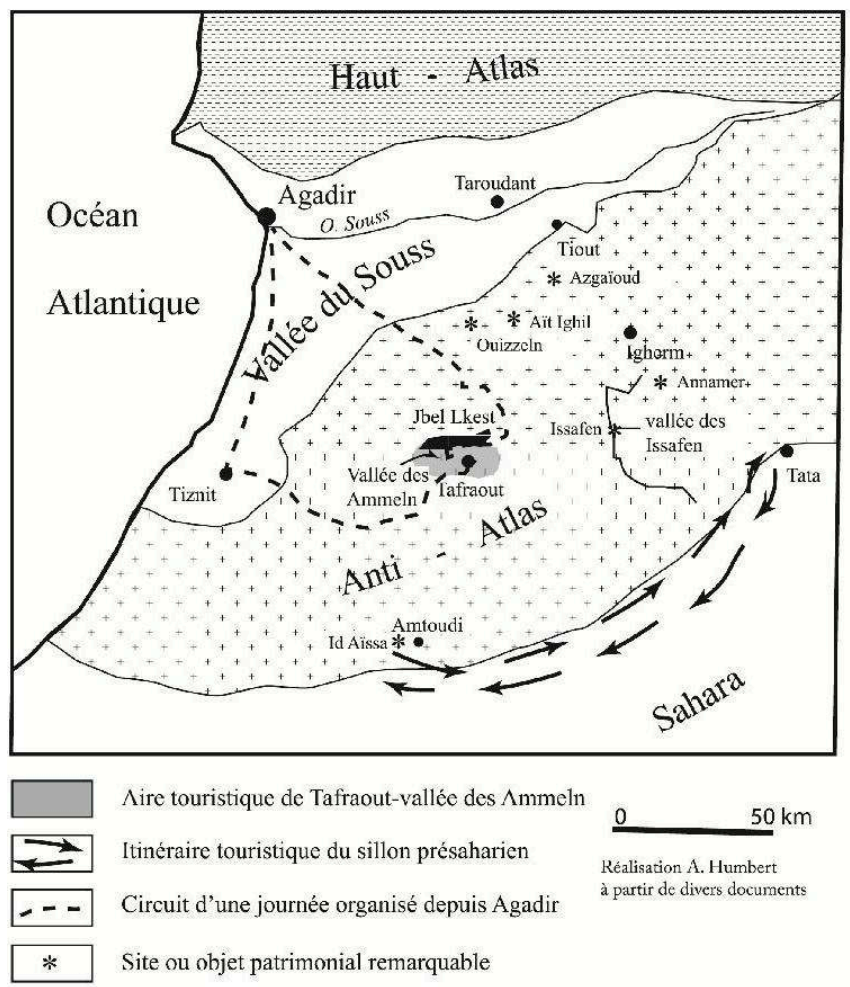

Réalisation personnelle 
Deux secteurs restreints de la montagne sont concernés par une relative activité touristique. Il s'agit d'une part, d'un foyer ancien et actif situé au cœur de la montagne qui bénéficie d'une ancienneté évidente, d'un environnement physique attractif et d'une communication difficile mais ancienne avec Agadir et la plaine du Souss : le petit foyer de Tafraoute et la vallée des Ammeln. La "mise en tourisme » de ce secteur doit sans doute beaucoup à la présence d'un gîte d'étape édifié dans les années 1940 et dont la réputation a été établie par une visite royale (fig. 4). Le second secteur de quelque importance a la forme linéaire d'un chapelet de ressources modestes qui s'étire sur environ $200 \mathrm{~km}$, dans un sillon présaharien, pourvu d'une route goudronnée stratégique, d'ouest en est, entre Tarjijt et Tata, et se prolonge ensuite, vers l'est, jusqu’à Foum Zguid.

Figure 4. Le gîte rural des « Amandiers » à Tafraoute (Tiznit).

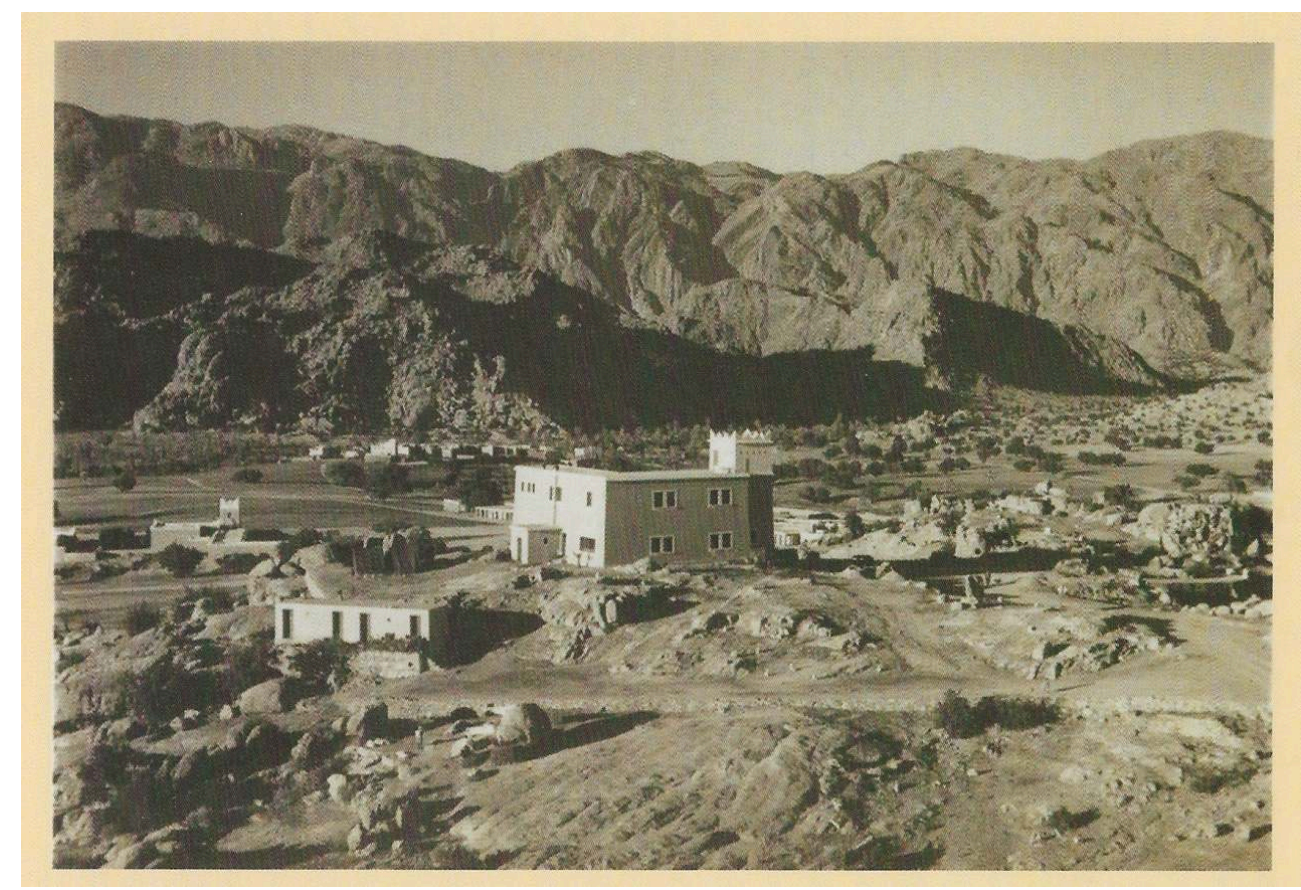

Au début des années 1940, cet établissement a été le germe du développement ultérieur de la seule concentration touristique de l'Anti-Atlas occidental. II a été agrandi et modernisé pour être aujourd'hui le plus grand hôtel de Tafraoute. (Humbert et Popp, 2016, p. 5).

Source : carte postale ancienne

Quelles sont les raisons de cette différence? Cela tient-t-il à une pacification plus tardive de la montagne? L'est de la chaîne est-il incomparablement plus riche culturellement que la partie occidentale ? La faiblesse relative d'Agadir par rapport à Marrakech en fait-il un réservoir insuffisant pour entraîner des flux significatifs ? Autant de questions - et sans doute à d'autres - auxquelles il convient de répondre pour apporter un début de réponse à ce déséquilibre. 


\section{L'Anti-Atlas occidental : forces et faiblesses}

\section{Des ressources culturelles indiscutables}

19 L'Anti-Atlas occidental, ne possède pas comme à l'est d'une chaîne de demeures aristocratiques faciles à transformer en maison d'hôtes, mais cette partie de la montagne atlasique a été très humanisée par une société ancienne d'agriculteurs qui ont laissé dans les paysages des formes passives - ou parfois encore actives - d'une grande valeur patrimoniale (Humbert, Popp, 2016).

Dans toute la chaîne et surtout sur le versant nord, les communautés paysannes ont modelé les versants en y édifiant des milliers de kilomètres de terrasses sèches étroites pour y installer des terroirs productifs (fig 5 ).

Figure 5. Versants de l'Anti-Atlas occidental près d'Azgaïoud (Taroudant) aménagés en innombrables terrasses " sèches".

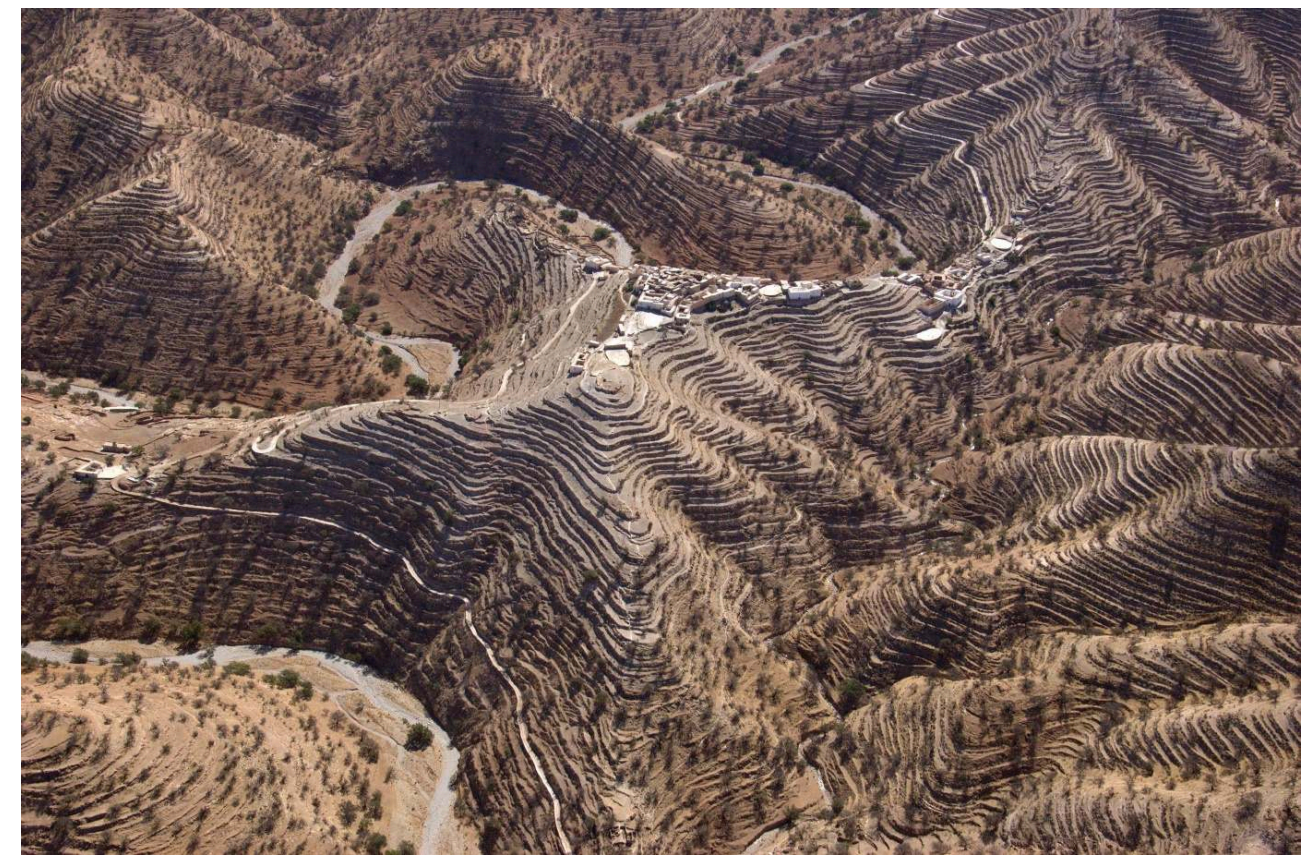

Cl. Humbert-Lemmel, 2009

21 Ces escaliers ubiquistes associés à diverses formes de récupération des eaux agricoles ont été récemment étudiés par un enfant de cette montagne (Ziyadi, 2013). Il a montré que toute une collection de formes patrimoniales étaient associées à l'aménagement des pentes, comme de belles aires à battre le grain, et un nombre considérable de citernes villageoises (matfia) (voir $\mathrm{n}^{\circ} 7$ de la fig. 8) destinées à recueillir, pendant les brèves périodes pluvieuses, l'eau nécessaire aux hommes et aux animaux domestiques. Sur le versant sud, dans un environnement déjà désertique, les paysans des oasis, au fond des vallées, ont acquis la science des aménagements parcellaires qui leur permettent de superposer les cultures et de distribuer l'eau nécessaire. Ils ont inventé des artefacts qui leur permettent de répartir équitablement l'eau - bien précieux et rare - au cours d'un cycle calendaire rigoureux, comme cette clepsydre appelée ici tanast (fig. 6) ou ce spectaculaire cadran solaire horizontal (fig. 7) (Humbert, 2006). 
Figure 6. Tanast du douar Annamer (Tata).

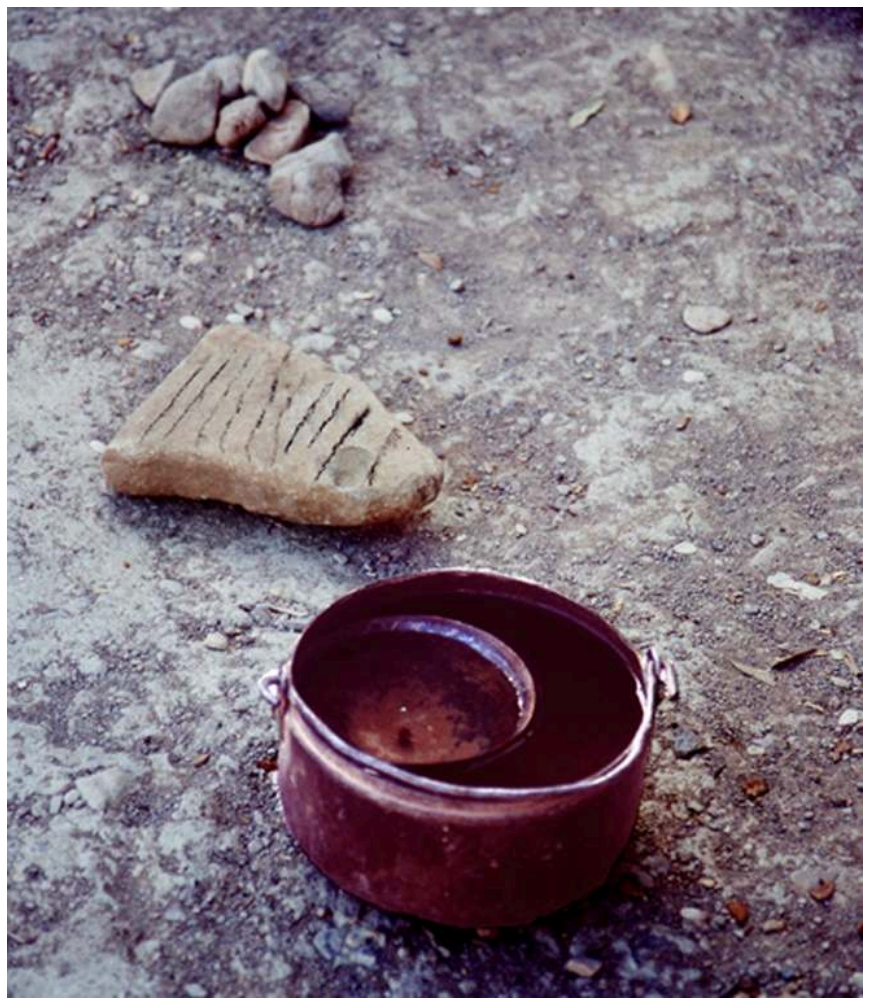

Cette clepsydre rudimentaire était d'un usage traditionnel très répandu. Le bol de cuivre (tanast), percé en son fond, s'enfonçait lentement (de 5 à 20 minutes) dans un réservoir fixe, ou mobile comme ici. Une comptabilité précise des tanassin immergées était faite par l'aiguadier (amazzal) à l'aide de 10 cailloux et d'un marquage sur une pierre plate à l'aide d'un charbon de bois.

Cl. Humbert, 1993

Figure 7. Cadran solaire à Issafen (province de Tata) (Cl. Humbert, 1997).

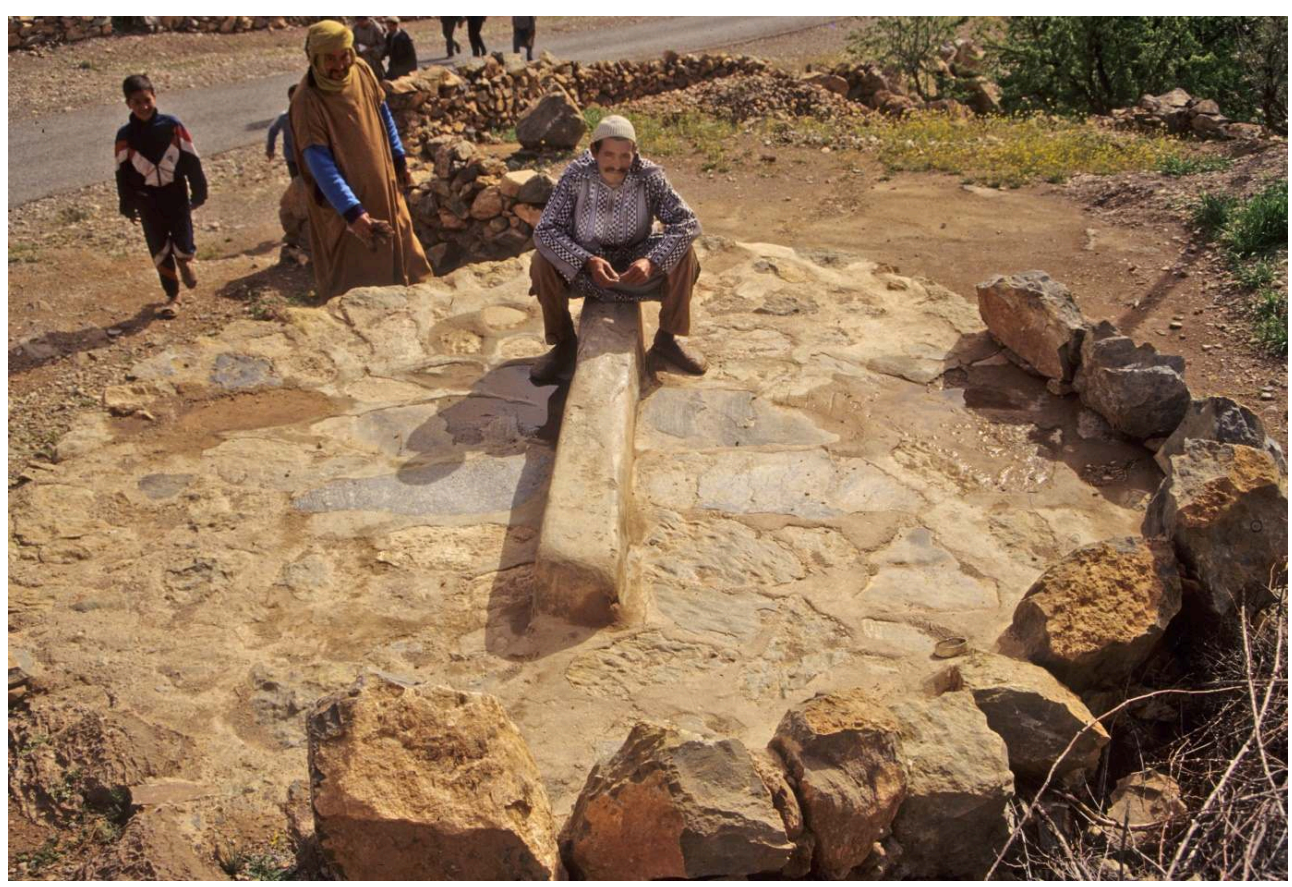

Une technique de mesure du tour d'eau dans les oasis de la vallée des Issafen 
Mais surtout, si l'Anti-Atlas occidental ne possède pas les somptueuses kasbahs de l'est, il offre un joyau architectural avec les igoudar (plur. de agadir). Les igoudar sont des greniers fortifiés collectifs, dont la construction s'étale peut-être sur un millier d'années ; cependant, les formes les plus classiques et les plus récentes remontent sans doute au XVIII ${ }^{\mathrm{e}}$ siècle. De nombreux auteurs se sont penchés sur ce phénomène (historiens, archéologues, architectes, géographes), ont mené les premières études (Montagne, 1929), en ont établi le plan (Jacques-Meunier, 1951), les ont étudiés en détail (Naji, 2006) ou en ont fait un inventaire quasi exhaustif (Popp, Aït Hamza \& El Fasskaoui, 2012). Si les formes les plus répandues sont assez simples, comme cet exemplaire observé dans la partie septentrionale et centrale de l'Atlas (fig. 8 et 9), beaucoup de ces greniers ont adopté des formes variées, signe souvent de leur ancienneté ou de leur polygénisme. Ces constructions qui peuvent rassembler plusieurs centaines de cases de stockage familial des grains et autres richesses sont aussi un patrimoine social car les greniers étaient des lieux de rassemblement d'une partie au moins de la communauté qui y avait une salle de prière, des citernes et parfois des boutiques, le tout sous la vigilance d'un gardien, l'amin qui en détenait - ou détient encore - la clé (fig. 10). Ces monuments impressionnants sont répartis dans toute la chaîne, jusqu'aux confins sahariens même si quelques secteurs, mystérieusement, en sont dépourvus.

Figure 8. L'agadir d'Aït Ighil (Taroudant) dans son environnement domestique proche.

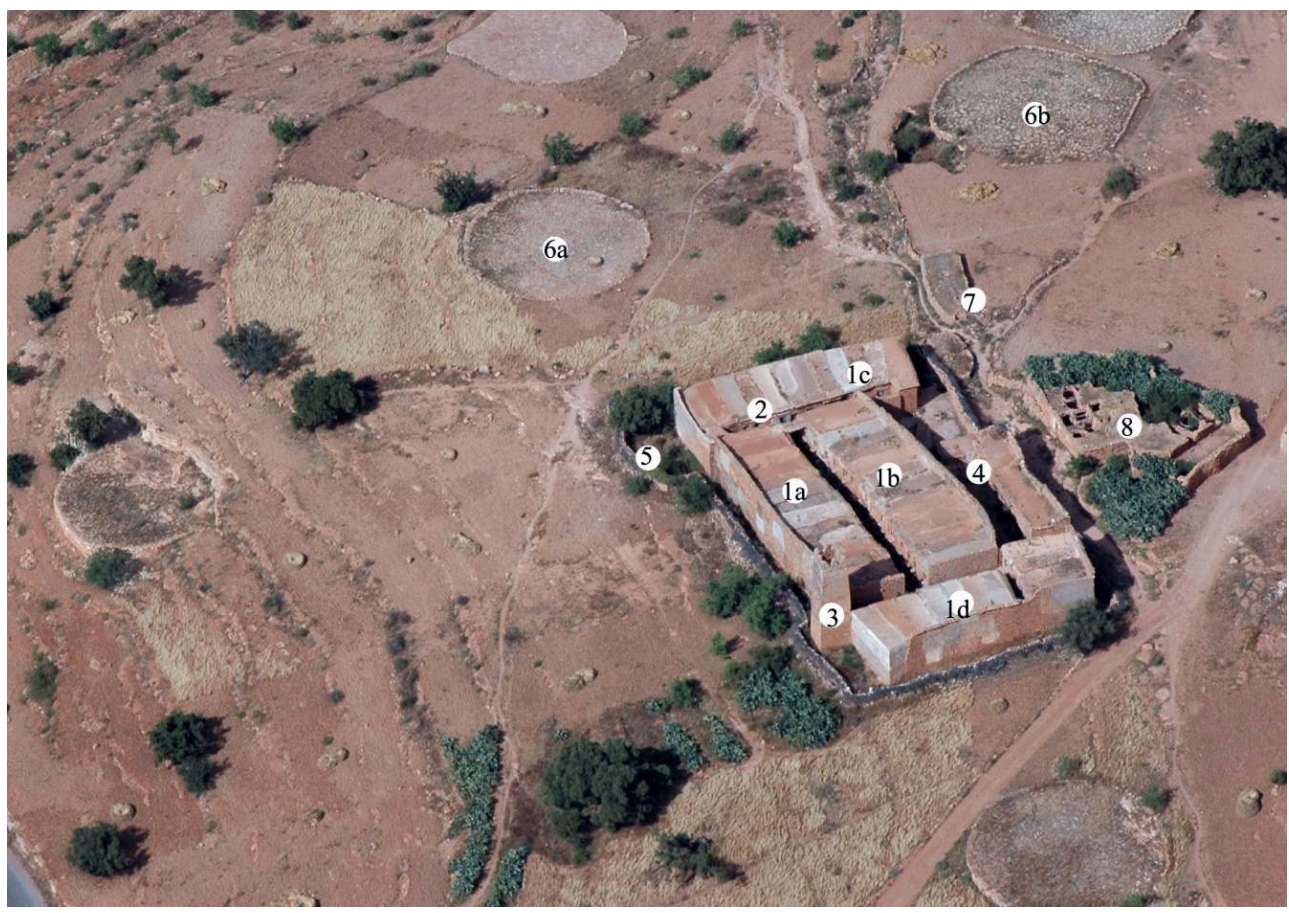

Cet agadir est « classique » avec ses deux travées principales (1a et 1b) augmentées de deux travées transversales complémentaires (1c et 1d). Si la gestion de l'agadir est collective, chaque famille est responsable de sa case dont la couverture diffère, ici, de sa voisine (2). Les igoudar possèdent généralement une ou plusieurs tours de vigie et de défense (borj) (3). Le grenier proprement-dit est précédé d'une cour commune, ici assez étroite (4) et d'une enceinte symbolique (5) que l'on ne transgresse qu'en commettant un sacrilège ; l'agadir est haram. L'environnement villageois proche est intéressant aussi avec ses aires à battre (6), sa citerne (7) et les ruines de sa petite mosquée rurale (8). Aït Ighil est un bon exemple de la concentration d'un patrimoine rural remarquable et facilement accessible par une route proche maintenant goudronnée.

Cl. Humbert-Renard, 2006 
Figure 9. Intérieur d'un grenier collectif avec les cases familiales.

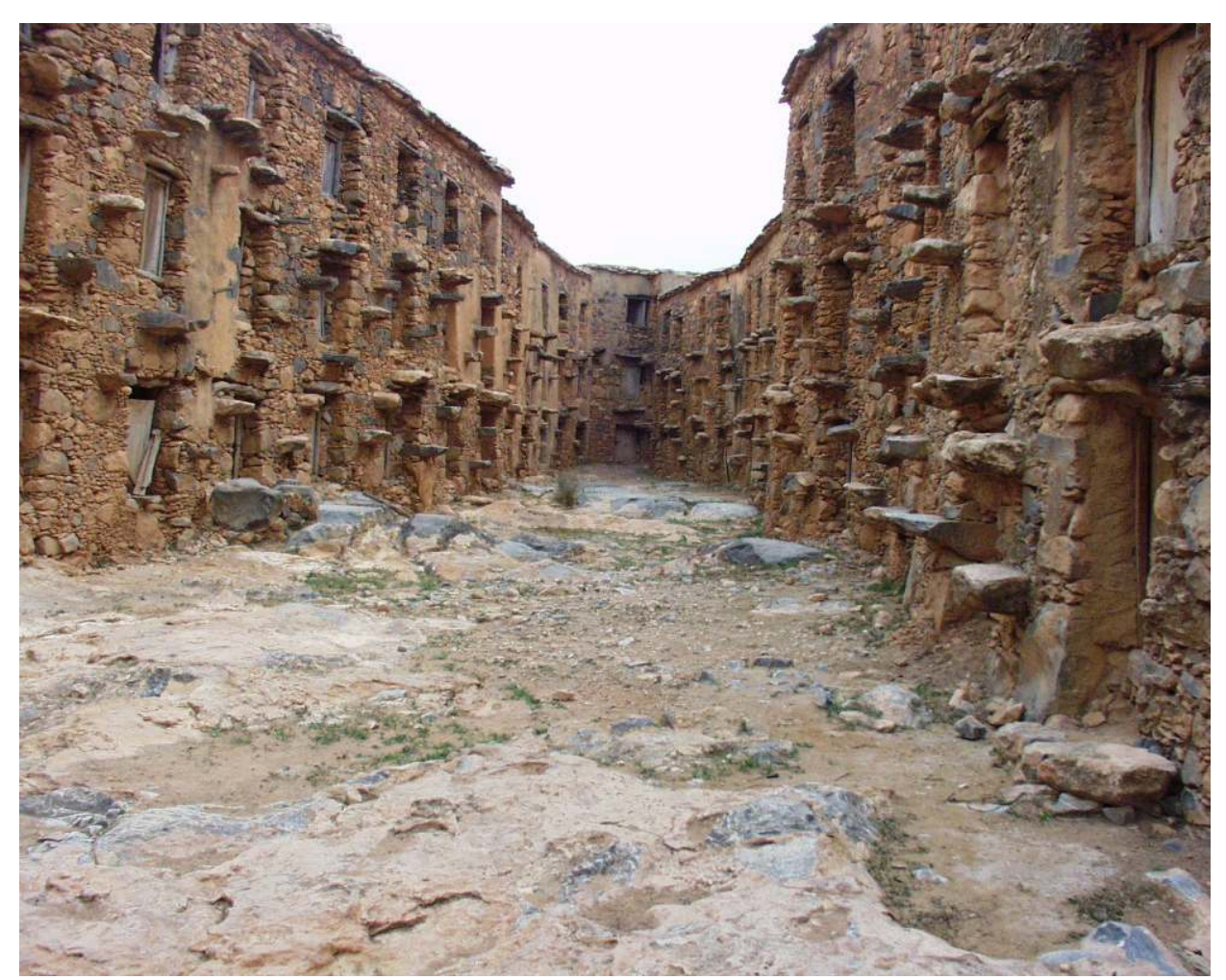

On y accède, ici, grâce à des dalles de pierre incluses dans la paroi principale.

Cl. Humbert, 2005 
Figure 10. Plan de l'agadir d'Oumsdikt (province de Taroudant).

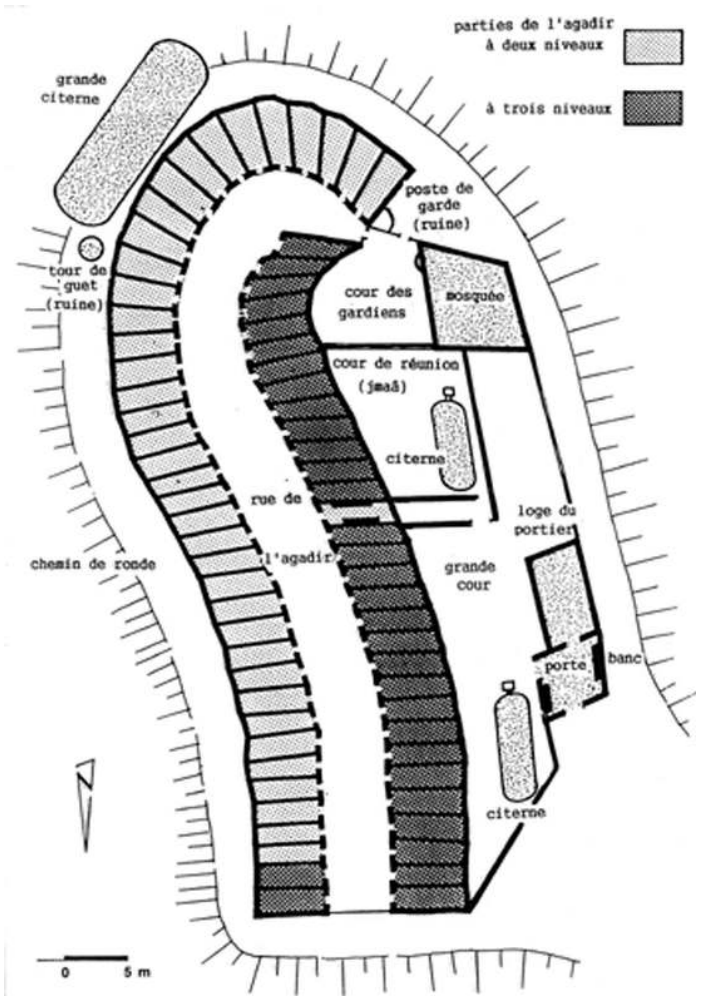

L'agadir n'est pas qu'un grenier collectif. C'est un édifice de la communauté qui, autrefois, remplissait une fonction sociale évidente avec ses va-et-vient et sa cour, à la fois lieu de prière, enceinte de réunions, atelier et boutique.

Humbert, 2012

Le patrimoine dispersé, de nature variée, possède un foisonnement favorisé par l'isolement conservateur même si ces campagnes ont été envahies par l'architecture obséquieuse d'émigrés « enrichis» revenus au bled pour y exposer leur réussite. Tout ne peut pas être mis en valeur pour être « mis en tourisme ». Les pièces maîtresses sont évidemment les igoudar, mais il est possible de faire vivre autour de quelques exemplaires bien choisis et bien conservés tout un complexe rural formé de nombreux éléments mineurs et de savoir-faire paysans; certains outils, des pratiques hydrauliques ou des objets de l'apiculture si répandue peuvent être réunis dans l'agadir, assez vaste pour cela. A proximité peut être aménagé un «vrai » terroir irrigué rendu vivant par des fellah(s) volontaires qui y trouveraient leur intérêt. Quelques tentatives sont observables dès maintenant, en particulier dans le sillon présaharien ${ }^{5}$ (Aït Hamza, Aït Saïd, 2013). Même si dans ces cas, toute la chaîne touristique n'est pas exploitée, et quelque imparfaites que soient ces expériences, elles sont cependant encourageantes (fig. 11 et 12). 
Figure 11. Amtoudi, vallée présaharienne (Province de Tata).

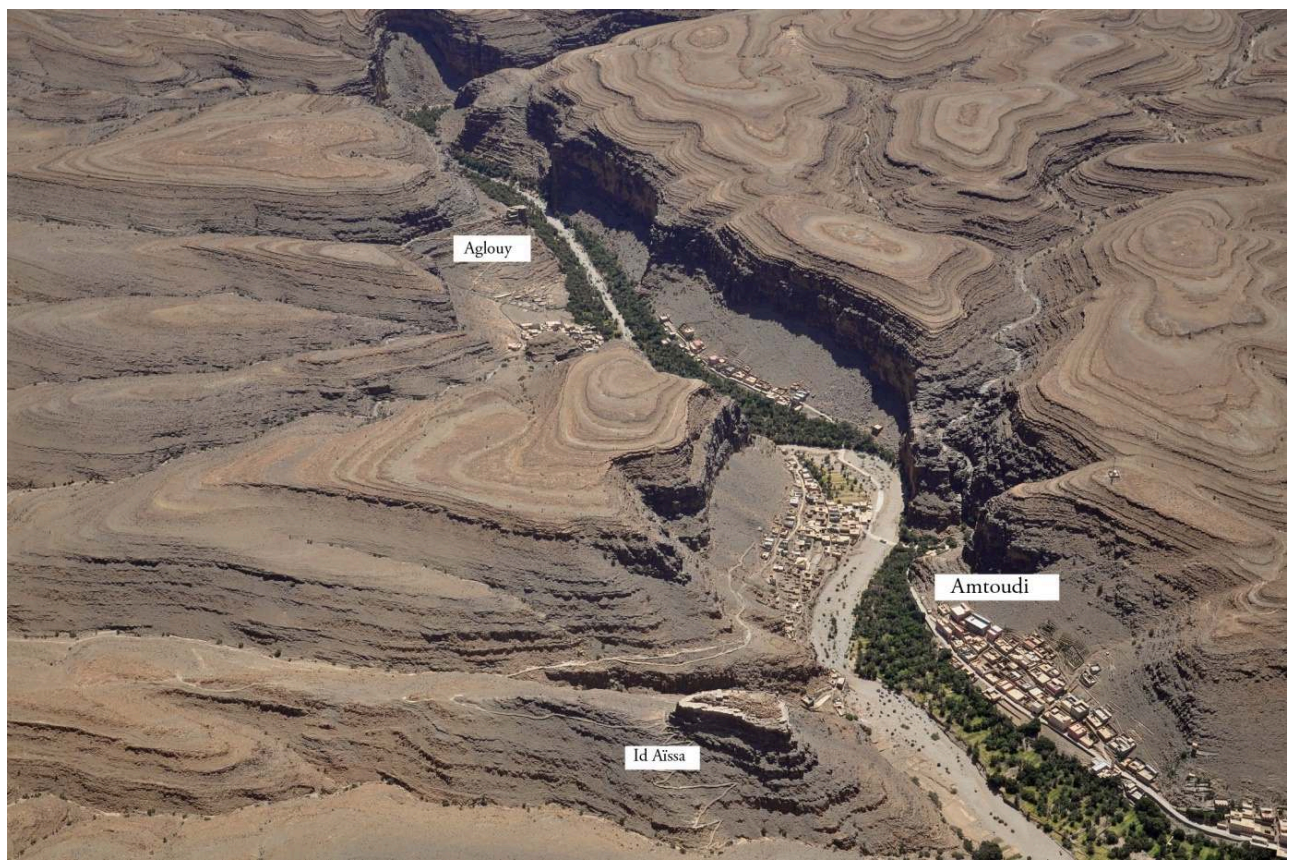

Sur le parcours du sillon présaharien, Amtoudi offre son oasis dans un cadre grandiose mais aussi deux igoudar qui surveillaient les razzias venues du sud et en préservaient les biens des oasiens.

Cl. Humbert et Popp, 2014

Figure 12. Rucher de l'agadir Id Aïssa à Amtoudi (Tata).

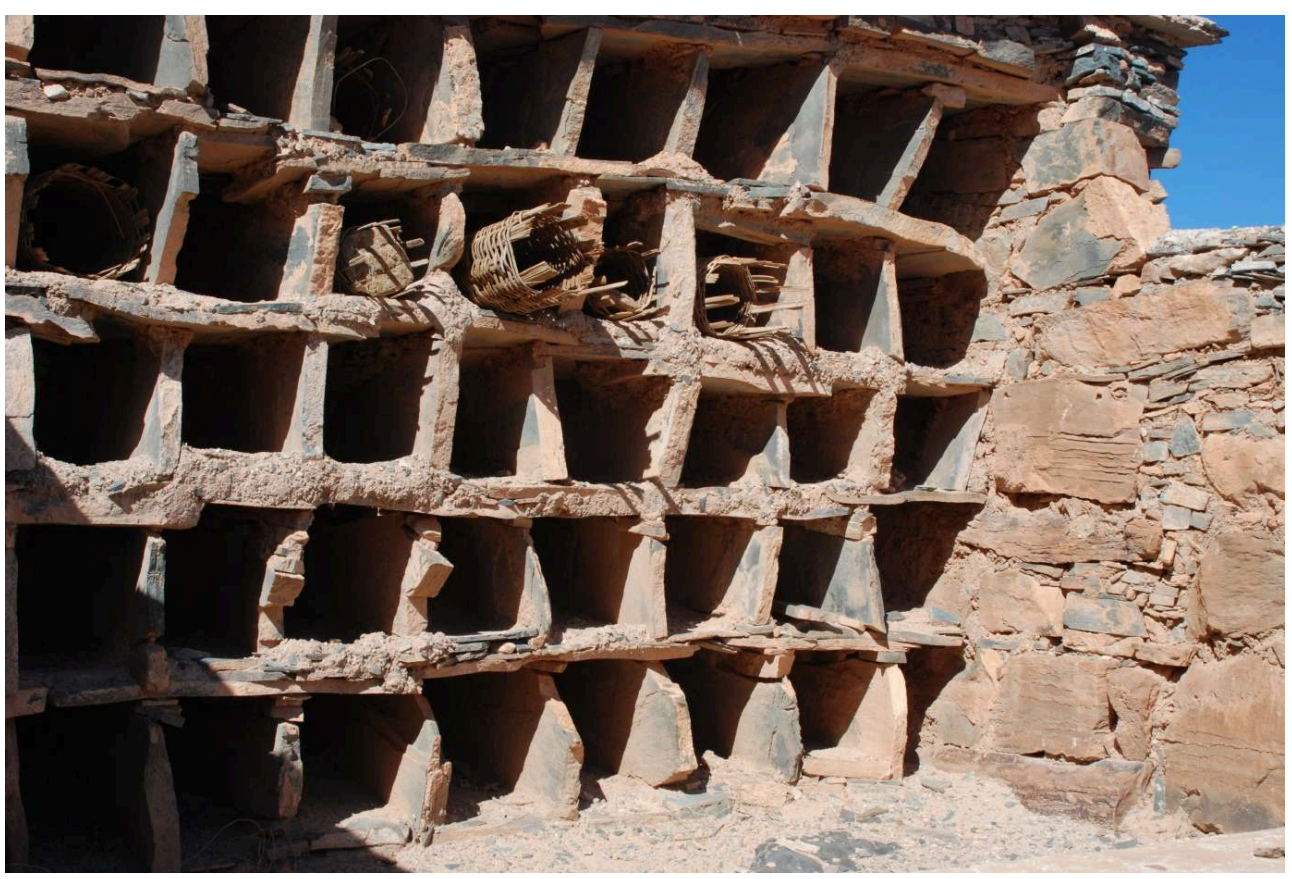

Les igoudar sont des édifices où peuvent être réunis de nombreux objets patrimoniaux des campagnes atlasiques. Ici, le rucher, in situ, témoigne de la vie sociale de l'agadir.

Cl. Humbert, 2013

Cette partie de l'Anti-Atlas possède donc une richesse patrimoniale indiscutable, probablement aussi abondante et aussi variée que dans la partie orientale. Pourquoi le 
tourisme culturel - et de nature - n'y a pas eu, jusqu'à maintenant, le même succès pour offrir une solution, au moins partielle à la déprise rurale? Les causes sont sans doute multiples. En voici quelques-unes.

\section{Des communications difficiles mais une ouverture rapide}

L'Anti-Atlas occidental, en raison de sa structure, ne se laisse pas facilement pénétrer. Longtemps, il n'a été traversé que par deux vieilles routes coloniales. L'une permettait de gagner Tafraoute, dans sa partie très occidentale ; l'autre de traverser la chaîne, plus à l'est, en passant par un poste militaire installé sur le col qui fait correspondre les deux versants : il s'agit de la bourgade d'Igherm. Pour autant, progressivement depuis la fin des années 1990, la circulation à l'intérieur de la montagne est devenue plus aisée et les routes goudronnées se sont multipliées à un rythme étonnant au cours de la dernière décennie, aussi bien dans le sens longitudinal que transversal. L'Anti-Atlas occidental est maintenant pénétrable.

\section{Une déficience des équipements d'hébergements touristiques}

Si la carte établie il y a quelques années (Popp et al., 2012) montre un déséquilibre certain, au profit de Tafraoute et d'un alignement présaharien, la situation n'a pas évoluée depuis de façon sensible : quelques maisons d'hôtes se sont ajoutées à celles existantes, souvent dans les mêmes secteurs et une «ville » étape comme Igherm n'a toujours pas d'hôtel digne de ce nom sur un itinéraire de quelque 200 kilomètres. Ceci est aussi le cas du long sillon, le seul aussi largement ouvert et amplement peuplé, celui des Issafen qui, après Igherm, conduit à Tata et au sillon pré-saharien. Cette vallée qui est un chapelet d'oasis, possède un riche patrimoine d'igoudar et d'artefacts ruraux de toute sorte. Cette vallée est loin d'être capable d'accueillir autant de touristes que celle du Dadès avec ses kasbahs. Même Tata, sur la grande rocade pré-saharienne du sud ne possède pas un équipement d'accueil digne de sa situation. Compte-tenu de la grande dispersion des objets patrimoniaux capables d'attirer les touristes, il semble bien que les structures légères, telles les maisons d'hôtes, capables d'accueillir quelques familles à la fois, soient mieux adaptées que les grosses structures hôtelières. Ce genre d'hébergement s'est développé, en concurrence avec l'hôtellerie dans le secteur de Tafraoute, en profitant de la réputation de l'aire touristique et des possibilités d'activités de nature au pied d'un des massifs les plus élevé de l'Anti-Atlas, le Jbel Lkest.

\section{Les obstacles à l'épanouissement d'un tourisme rural}

Les exemples encourageants observés çà et là dans l'Anti-Atlas ne sauraient masquer un certain nombre de difficultés qui s'ajoutent à la grande dispersion du patrimoine et à la médiocrité de l'équipement d'accueil.

Le premier tient à la propriété collective des igoudar, contrairement à celui des kasbahs de la partie orientale. En effet, les greniers collectifs appartiennent à la communauté dirigée par le conseil qu'est la jmâa. Il faut un accord collectif pour entretenir et exploiter l'édifice, ce qui ne va pas de soi. Même quand l'exploitation est confiée à un particulier - vieux fellah ou ancien amin -, les jalousies sont inévitables et provoquent, parfois, l'abandon de l'exploitation. La visite de nombreux igoudar, aussi bien par $\mathrm{H}$. 
Popp et B. El Fasskaoui que par l'auteur de cet article, ont conduit à identifier cette difficulté de l'exploitation des igoudar par le tourisme, en raison de leur propriété collective, revendiquée toujours par le jmâa. La gestion de ces édifices se complique encore quand ils appartiennent à plusieurs communautés villageoises ce qui est fréquent car les igoudar servaient souvent d'entrepôt à plusieurs villages (fig. 13).

Figure 13. Agadir Aït Ouizzeln (province Chtouka-Aït-Baha), un des plus grands de l'Anti-Atlas (260 cases).

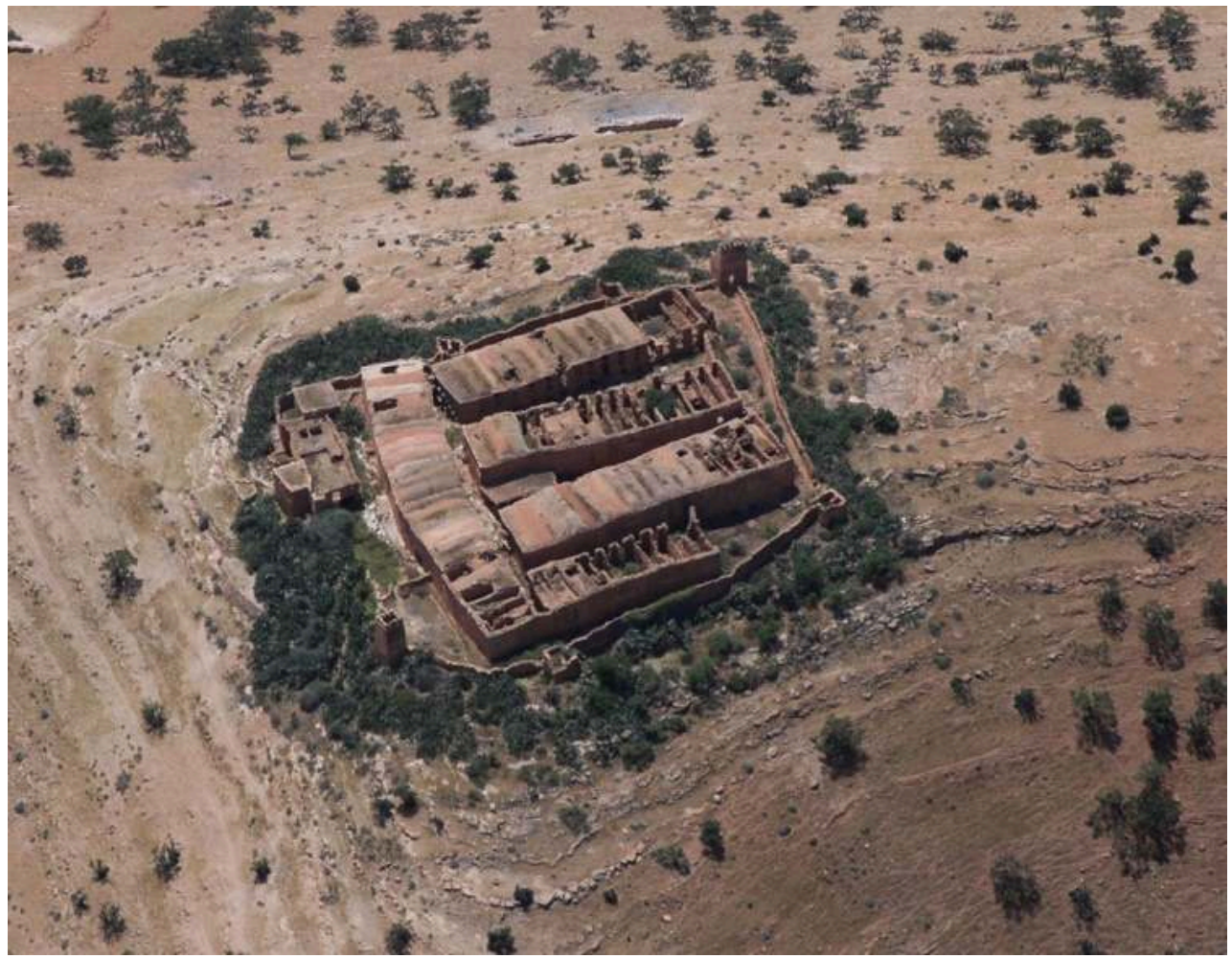

C'est un bon exemple du danger qui menace le patrimoine construit des campagnes s'il n'attire pas des flux suffisants de touristes.

Cl. Humbert-Renard, 2006

Un autre point important est souvent sous-estimé dans la prise en charge des touristes qui viennent pour découvrir dans un environnement culturel rural qui n'est pas - ou n'est plus - le leur. Ce n'est pas l'information culturelle véritable mais seulement l'ambiance culturelle que des guides mal formés proposent généralement aux groupes de visiteurs. Malgré un effort officiel fait pour proposer des guides agréés et marginaliser les «faux» guides qui n'ont guère que leur sympathie à offrir et quelquefois une connaissance pratique du terrain, le niveau de la médiation culturelle reste très médiocre. En 2018, la fédération marocaine des guides protestait contre l'attribution à quelque 1100 candidats de la qualification aux fonctions de Guides des Espaces Naturels «malgré leur illettrisme " ${ }^{6}$. Même s'il est possible de voir dans cette protestation la crainte corporatiste d'une concurrence, il est évident que la rencontre fréquente avec ces "médiateurs » et les échanges avec eux, atteste souvent de leur indifférence pour les objets et les pratiques culturelles. Les Guides des Espaces Naturels (GEN) ont certes reçu une formation mais celle-ci porte davantage sur la gestion matérielle des groupes que sur une véritable information sur les pratiques agraires et sur tout l'environnement matériel de celui-ci. Ces guides officiels sont des urbains de la 
plaine du Souss qui ont perdu, depuis longtemps, le contact réel avec la campagne et qui sont, le plus souvent ignorants de ce qu'ils devraient expliquer aux touristes. Cette indigence culturelle apparaît d'ailleurs à la lecture des programmes publiés par les officines gadiries qui traduisent plus leur intérêt commercial que leur souci culturel. Ces médiateurs culturels devraient avoir une solide formation de naturalistes, d'historiens et de géographes. Ce qui n'est évidemment pas le cas. Il y a une quinzaine d'années, dans le cadre d'une coopération interuniversitaire avec l'Université d'Agadir, avait été envisagée la création d'une section réunissant les meilleurs étudiants de géographie, d'histoire et de sciences de la Terre, répartis en différents groupes linguistiques, pour former de solides médiateurs culturels. Malgré une bonne volonté évidente le projet n'a pu voir le jour, sans doute pour diverses raisons et peut-être l'opposition d'une école locale de tourisme.

Les touristes intéressés en sont réduits à organiser eux-mêmes les visites vers les lieux les plus réputés, comme le site de Tafraoute et ses environs ou quelques centaines de camping-cars s'entassent dans quelques campings ou, en désordre, sur quelque terrain vague (fig. 14).

Figure 14. Caravaning anarchique en bordure de l'agglomération touristique de Tafraoute.

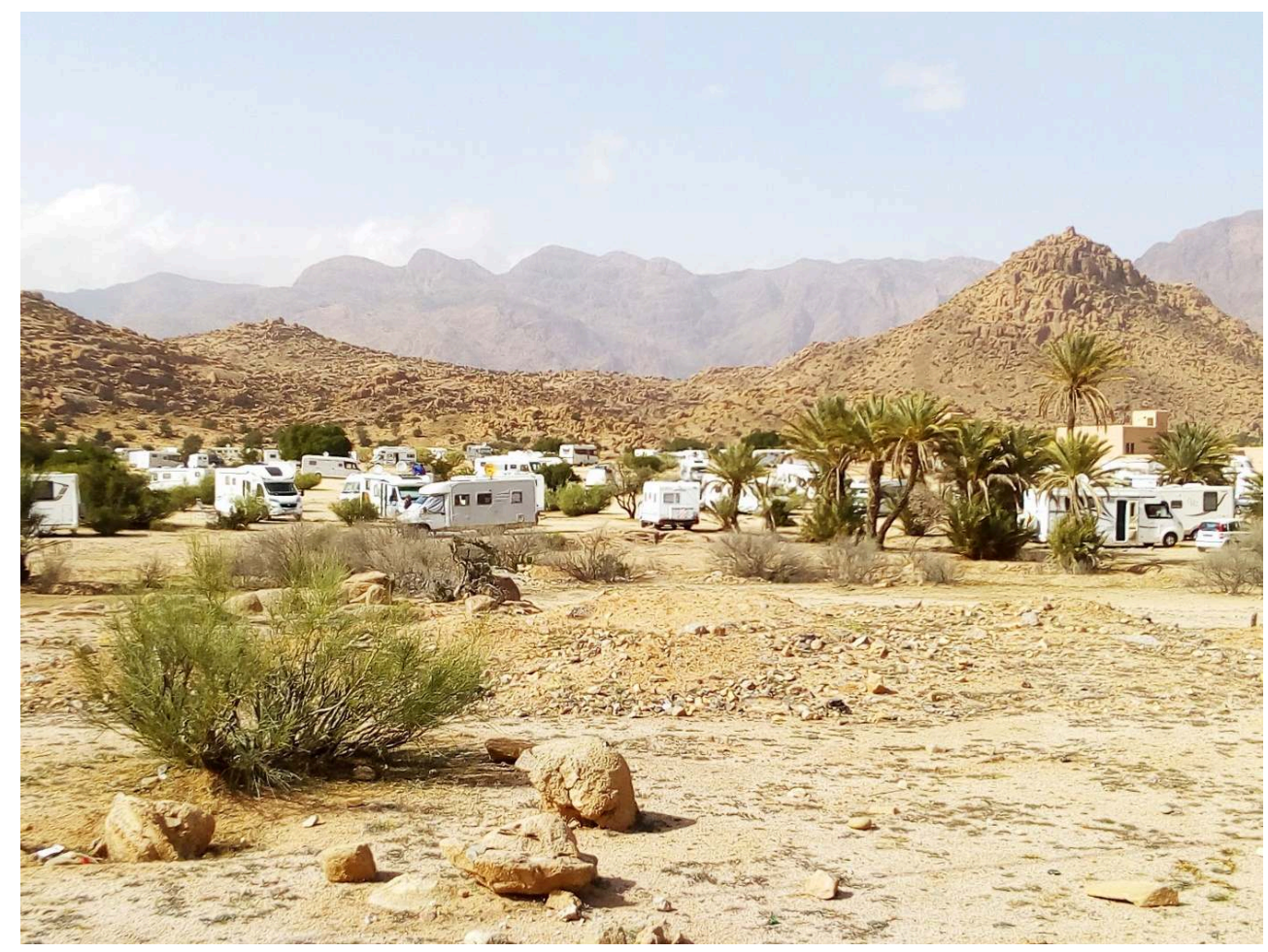

Cl. Humbert, 2019

31 Les visites les plus systématiquement organisées sont le fait de quelques agences gadiries qui proposent des sorties d'une journée avec la complicité - et l'assentiment des tours opérateurs qui possèdent ou contrôlent les chaînes hôtelières de la station balnéaire. Les groupes sont conduits vers la vieille capitale saadienne, Taroudannt, dans la plaine du Souss à $80 \mathrm{~km}$ seulement d'Agadir. Ce n'est pas très loin et l'on peut laisser les touristes flâner dans les souk(s), les promener jusqu'à « l'oasis » de Tiout à seulement $35 \mathrm{~km}$ de Taroudannt, et leur faire visiter une coopérative d'huile d'argan pour leur permettre d'acheter des produits dérivés. Les mêmes officines réussissent la 
performance de proposer une seule journée d'excursion dans l'Anti-Atlas, pour les faire passer par Tafraoute - le sanctuaire du tourisme dans l'Anti-Atlas occidental - avec un retour par Tiznit et le sud de la plaine du Souss. C'est une virée harassante de 12 heures et $400 \mathrm{~km}$ ! Le programme de cette journée, probablement traduit de l'anglais, donne les horaires précis et les principales haltes, y compris les pauses-café (cafés ou thés payants!). Le voyage commence à $6 \mathrm{~h} 30$, heure à laquelle les excursionnistes sont «ramassés» à l'hôtel. Sur le parcours, les touristes verront «des paysages désertiques ", " des pics déchiquetés ", des "rochers géants ", " des canyons aux murs rouges", des "huttes de boue (sic) des berbères » et " des arbres producteurs de pétrole (sic) d'argan ». Cette visite est une course effrénée. A 11 heures, la voiture climatisée arrive dans la vallée des Ammeln où l'on montre aux touristes les villages au pied du Jbel Lkest (fig. 15), puis l'on gagne Tafraoute, tout près de là, où une halte de $2 \mathrm{~h} 15$ est prévue. C'est le temps estimé nécessaire pour déjeuner (de préférence à l'hôtel des Amandiers, pour $15 €$ ou $120 \mathrm{Dh}$ ) et visiter le "souk», c'est-à-dire tous les commerces qui proposent les productions «locales", notamment tous les produits cosmétiques dérivés de l'huile d'argan. Départ pour Tiznit à $14 \mathrm{~h} 15$ précises. Arrivée à $16 \mathrm{~h} 30$ après un long parcours de montagne. Le groupe fatigué dispose d'une heure pour voir les remparts de la ville et surtout visiter le quartier des bijoutiers. A la pratique dominante de la découverte s'ajoute celle du shopping (Renard-Grandmontagne, 2017). Le départ est donné à $17 \mathrm{~h} 30$ pour un retour à l'hôtel vers $19 \mathrm{~h} 30$ ! Il est évident que les maîtres du tourisme mondialisé ne souhaitent pas perdre une clientèle "empackagée ", avec occupation de six ou sept nuits dans la chambre d'un grand hôtel gadiri. Le circuit proposé mériterait au moins deux jours. C'est totalement impossible : l'excursion ne peut être qu'un supplément à un programme vendu clé en main. On voit là comment les tours opérateurs peuvent freiner l'accueil et donc le développement touristique dans l'Atlas occidental. 
Figure 15. La vallée des Ammeln (Tiznit).

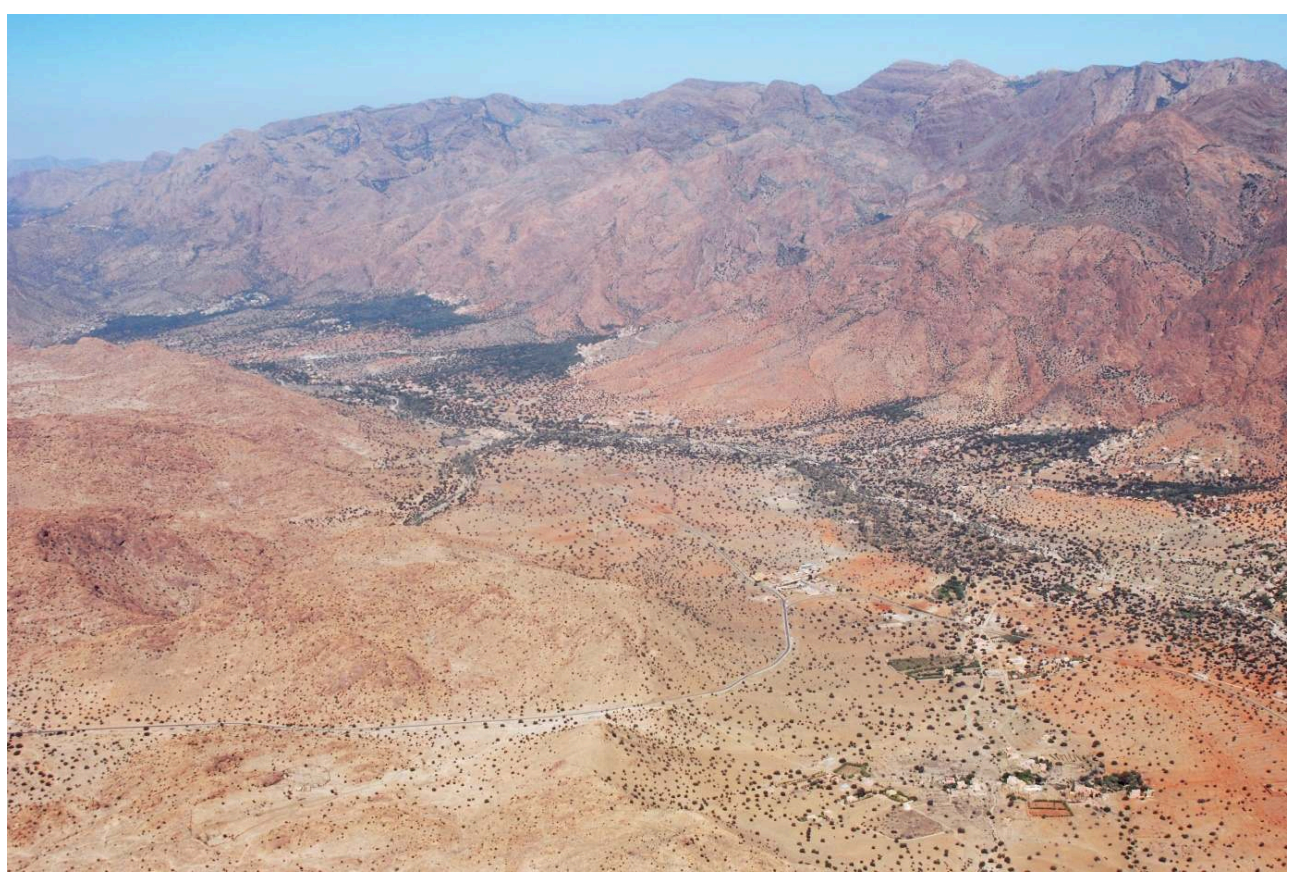

Cette longue dépression au pied d'un des massifs les plus élevés de l'Anti-Atlas, le Jbel Lkest (2 360 $\mathrm{m}$ ) est tout proche de Tafraoute et a profité de cette proximité. Les touristes amateurs de culture populaire et de nature ne peuvent que s'y intéresser. Les agences gadiries la placent dans leur tourmarathon d'une journée.

Cl. Humbert-Popp, 2014

Le patrimoine culturel de l'Anti-Atlas est avant tout un patrimoine rural, fait de pratiques séculaires, d'objets et d'outils domestiques, d'une architecture vernaculaire et surtout des pièces architecturales majeures que sont les igoudar. L'indigence touristique a sans doute des raisons diverses. Les causes majeures viennent d'être exposées. Rappelons simplement, dans ce tableau, les atouts et handicaps énumérés et repérés ; il n'est sans doute pas exhaustif.

Tableau 2. Atouts et handicaps pour le tourisme rural de l'Anti-Atlas occidental.

\begin{tabular}{|l|l|}
\hline Atouts & Handicaps \\
\hline - Grande variété de paysages & - Compacité du relief \\
- Grande richesse du patrimoine rural naturel et & - Peu de forces vives dans une population âgée et \\
culturel & féminine \\
- Réseau maintenant étoffé de routes goudronnées & - Manque d'équipements de standing international \\
- Proximité d'un pôle touristique majeur (Agadir) & - Rivalités au sein des communautés pour la gestion \\
- Faible niveau d'insécurité au cours des dernières & - Médiocrité des médiateurs culturels actuels (GEN) \\
décennies & \\
- Couverture très large de la téléphonie mobile et du & - Accaparement du tourisme par les tours opérateurs \\
web. & \\
& - Faible efficacité de la gouvernance publique ou \\
\end{tabular}

Tableau synthétique établi par l'auteur 


\section{Conclusion} ont encouragé, à certaines époques, un tourisme balnéaire attirant des flux plus importants. Dès avant la dernière guerre, le Sud pacifié et une habile exploitation du patrimoine construit ont attiré les touristes vers les sillons ouverts autour de l'AntiAtlas oriental. La partie occidentale de cette chaîne n'a pas bénéficié du même intérêt, si ce n'est dans quelques zones privilégiées, alors qu'elle possède pourtant des atouts patrimoniaux indiscutables et une ouverture plus récente favorisée par le revêtement de nombreuses pistes et une couverture remarquable par les moyens modernes de communication. Il n'est pas sûr cependant, que la présence proche d'Agadir, pôle balnéaire de réputation internationale (Berriane, 2002), n'ait pas un effet plus stérilisant que stimulant sur le développement touristique de la montagne voisine. Le même auteur et une collaboratrice (Berriane, Nakhli, 2011) ont montré, qu'au contraire, le pôle touristique d'Essaouira a un dense réseau d'hébergements autour de la ville. Mais cette mise en tourisme de la campagne s'effectue dans un rayon de 15 à 20 $\mathrm{km}$. Les premiers sites de l'Anti-Atlas occidental sont à $75 \mathrm{~km}$ !

Nos observations ont porté tant sur le déséquilibre ou l'indigence touristique que sur l'ouverture progressive de la montagne ou que sur la «mise en tourisme» de seulement une petite partie de la richesse patrimoniale des campagnes. Cette étude, fondée avant tout sur des enquêtes informelles et une connaissance intime de cet espace montagnard plus que sur des statistiques, souvent fragmentaires et sujettes à caution, sera certainement à reprendre pour étudier les conséquences sur le tourisme de l'ouverture quasi généralisée de la montagne. Il est probable, notamment, que la couverture de cet espace par le réseau Internet profitera aux entrepreneurs individuels qui pourront contourner ainsi les tours-opérateurs qui agissent depuis Agadir. Il reste que le riche patrimoine, produit par une étonnante culture paysanne, est très dispersé et menacé par la modernité. Raison de plus pour que des médiateurs culturels, cultivés et correctement formés, sachent le mettre en valeur et le protéger.

\section{BIBLIOGRAPHIE}

AÏT HAMZA M., AÏT SAÏD H. (2013), « Mise en tourisme des greniers collectifs : cas d'Id Aïssa et Aglouy, in AÏT HAMZA M., H. POPP H. (éd.), Les igoudar : un patrimoine culturel à valoriser, Rabat, Publication de l'Institut Royal de la Culture Amazighe, série colloques et séminaires, 30 , pp. 253-263.

BERRIANE M. (2002), « La Souss-Massa et ses prolongements » in TROIN et al., Le Maroc. Régions, pays, territoires, Paris, Maisonneuve et Larose, pp. 295-324. 
BERRIANE M., NAKHLI S. (2011), « En marge des grands chantiers touristiques mondialisés, l'émergence de territoires touristiques "informels" et leurs connexion directe avec le système monde. Le cas de l'arrière-pays d'Essaouira, au Maroc », Méditerranée, 116, pp. 115-122.

BIERNERT U. (1998), Wüstentourismus in Sudmarokko. Das Beispiel des Tafilalt, Maghreb Studien, H11, Passau, L.I.S. Verlag.

BOUAOUINATE A., BECKEDORF A.S. \& POPP H. (2008), « Tourisme de masse et crue catastrophique dans le désert. La fin de la durabilité et du cycle touristique dans l'erg Chebbi (Sud du Maroc) estelle atteinte? ", in POPP H., Les pays du Maghreb. Contribution à la géographie humaine allemande, Bayreuth, Deusche Geselleschaft für Geographie

EL FASSKAOUI B., CAGERMEIER A. (2014), Patrimoine et tourisme culturel au Maroc. Actes du colloque maroco-allemand, Meknès, 2014, Meknès, Faculté des Lettres et Sciences Humaines, Université Moulay Ismaïl.

GAGNOL L., LANDEL P.-A. (2016), « Psammotourisme. Le sable au désert comme expérence et ressource touristique spécifique », Via, http://journals.openedition.org/viatourism/1364, DOI : https://doi.org/10.4000/viatourism.1364 (mis en ligne le $1^{\text {er }}$ décembre 2016, consulté le 20 octobre 2020).

HUMBERT A. (2003), « L’Anti-Atlas : une montagne paysanne moribonde ? », in RIEUTORT L. (coord.), Crises et mutations des agricultures de montagne, Clermont-Ferrand, Université Blaise Pascal, pp. 61-72.

HUMBERT A. (2006), « Comment mesure-t-on l'eau d'irrigation dans les communautés traditionnelles du Maroc méridional ? ", in CRESSIER P. (éd.), La maîtrise de l'eau en al-Andalus. Paysages, pratiques et techniques, Madrid, col. de la Casa de Velázquez, pp. 313-325.

HUMBERT A. (2009), « Potencialidades para un turismo sostenible en Marruecos meridional », Geographicalia, 2, pp. 5-36.

HUMBERT A. (2013), « L'agadir des Issendalène à Oumsdikt : un patrimoine en ruines », in AÏT HAMZAET M., POPP H. (éd.), Les igoudar : un patrimoine culturel à valoriser, Rabat, Publication de l'Institut Royal de la Culture Amazighe, série colloques et séminaires, 30, pp. 155-173.

HUMBERT A., POPP H. (2016), L'Anti-Atlas, une montagne marocaine méconnue. Découverte d'un riche patrimoine, Rabat, Institut Royal de la Culture Amazighe, $238 \mathrm{p}$.

JACQUES-MEUNIÉ D. (1951), Greniers citadelles au Maroc, Paris, Institut des Hautes Études Marocaines.

MARZOUK A. (2012), «Ifrane (Moyen Atlas) : d'un centre colonial de villégiature à un chef-lieu de province », in POPP H., AÏT HAMZA M., L'héritage colonial du Maroc, Bayreuth, Naturwissenschaftliche Gesellschaft Bayreuth, pp. 267-288.

MONTAGNE R. (1929), « Un magasin collectif de l'Anti-Atlas : l'agadir des Ikounka », Hespéris, 6, 2-3, pp. 145-266.

NAJI S. (2006), Les greniers collectifs de l'Atlas. Patrimoines du Sud marocain, Aix-en-Provence, Édisud, coll. Monde méditerranéen.

OUJAMÂA A. (2012), « Le tourisme intérieur français dans la période du Protectorat au Maroc, précurseur et initiateur du tourisme culturel ? Cas du Sud intérieur : la province de Ouarzazate » in POPP H., AÏT HAMZA M., L'héritage colonial du Maroc, Bayreuth, Naturwissenschaftliche Gesellschaft Bayreuth, pp. 289-306. 
POPP H. (2008), «La 'Route des Kasbahs' : un produit touristique du Maroc présaharien », in POPP H. (coord.), Les pays du Maghreb. Contribution à la géographie humaine allemande, Bayreuth, Deusche Geselleschaft für Geographie.

POPP H., AÏT HAMZA M. (2004), Randonnée culturelle dans le Djebel Sagho (Maroc du Sud), Bayreuth, Naturwissenschaftliche Gesellschaft Bayreuth.

POPP H., AÏT HAMZA M. (éd.) (2012), L’héritage colonial du Maroc, Bayreuth, Naturwissenschaftliche Gesellschaft Bayreuth, 340 p.

POPP H., El FASSKAOUI B. (2017), La route des kasbahs, Peterberg, Michael Imhof Verlag (ouvrage trilingue : allemand, français, anglais), $400 \mathrm{p}$.

POPP H., El FASSKAOUI B. \& AÏT HAMZA M. (2012), Carte touristique de l'Anti-Atlas occidental, Bayreuth, Naturwissenschaftliche Gesellschaft Bayreuth.

POPP H., AÏT HAMZA M. \& EL FASSKAOUI B. (2011), Les agadirs de l'Anti-Atlas occidental. Atlas illustré d'un patrimoine culturel du Sud marocain, Bayreuth, Naturwissenschafliche Gesellschaft Bayreuth, $500 \mathrm{p}$.

RENARD-GRANDMONTAGNE C. (2017), « Tourisme, loisirs et commerce : une « fertilisation croisée ", in FAGNONI E., Les espaces touristique et des loisirs, A. Colin, coll. Horizon, pp. 258-268.

SCHERLE N. (2012), « Les clichés et les stéréotypes de la période coloniale dans les guides de voyage touristiques allemands sur le Maroc et leur persistance jusqu'à nos jours ", in POPP H., AÏT HAMZA M., L'héritage colonial du Maroc, Bayreuth, Naturwissenschaftliche Gesellschaft Bayreuth, pp. 307-318.

TROIN J.-F. et al. (2002), Le Maroc. Régions, pays, territoires, Paris, Maisoneuve et Larose.

WRAGE W. (1967), Die Straße der Kasbahs, Unter der Berbern Südmarokkos, Radebeul, Neumann Verlag, $264 \mathrm{p}$.

ZIYADI M. (2013), Vie rurale et aménagement des pentes dans l'Anti-Atlas occidental, Maroc, Nancy, PUN-Éditions Universitaires de Lorraine, 233 p.

\section{NOTES}

1. Les informations concernant la fréquentation touristique ainsi que son impact économique sont consultables sur le site marocain du Ministère du Tourisme, de l'Artisanat, du Transport aérien et de l'Économie sociale. Le trafic des aéroports est communiqué par l'Office National des Aéroports du Maroc.

2. C'est-à-dire les touristes étrangers et les « Marocains Résidents à l'Étranger » (MRE).

3. Les kasbahs sont des maisons fortes édifiées pour les membres d'une certaine aristocratie politique et sociale de l'entourage du glaoui de Marrakech qui, au XIX siècle et au début de la colonisation, régnait sur cette région. Une des plus fameuses est une des kasbahs que le glaoui lui-même s'était fait construire sur le versant sud du HautAtlas, à Télouet. Ces maisons spacieuses et isolées ont été souvent transformées en hôtel ou maisons d'hôtes par leurs propriétaires actuels.

4. Office National des Aéroports (Maroc), Communiqué de presse $n^{\circ} 01 / 2019$.

5. L'oasis présaharienne d'Amtoudi, installée au fond de gorges, est dominée par deux magnifiques igoudar, bien conservés ou restaurés qui sont ouverts aux touristes qui acceptent de parcourir un chemin muletier long et raide. A leur pied, l'oasis offre un 
paysage saharien que les autochtones acceptent de présenter et parfois de commenter. Un hôtel, quelques maisons d'hôtes, et un terrain de camping-caravanning, facilitent le séjour, pour quelques jours, des touristes itinérants.

6. Voir l'article de Wiam Markhouss (2018) «Pourquoi la fédération des guides s'oppose à l'ouverture de la profession ", La Vieeco [en ligne] du 18 mars 2018.

\section{RÉSUMÉS}

Le tourisme culturel marocain est ancien; il remonte à l'époque du Protectorat, dès les années 1920. Il a privilégié d'abord les villes dites impériales avant de s'étendre au sud-est dans l'AntiAtlas oriental. L'Anti-Atlas occidental, pourtant doté d'une richesse patrimoniale certaine dans un environnement rural et agraire, n'a pas connu jusqu'à maintenant le même engouement, en dépit de la proximité d'Agadir, pôle touristique majeur. L'article tente d'expliquer le retard de la « mise en tourisme » de cette partie des chaînes atlasiques et présente aussi des exemples de sites et d'objets patrimoniaux susceptibles d'attirer les touristes. Alors que cette partie de l'Atlas n'a intéressé que récemment quelques géographes marocains ou étrangers, cet article s'appuie sur ces quelques recherches et surtout sur les observations ponctuelles et nombreuses effectuées par l'auteur, au cours des deux ou trois décennies passées, jusqu'en 1999.

Moroccan cultural toursim is an old custom; it dates back to the Protectorate era, from the 1920s. It first favored the so-called imperial cities before expanding to the south-east, in the Oriental Anti-Atlas. The Western Anti-Atlas yet endowed with a certain wealth of heritage, in a rural and agrarian environment, has not known, until now, the same craze, despite the proximity of Agadir, major tourist center. The article tries to explain this delay in the tourism development of this part of the Atlas chains and also presents examples of heritage sites and objects likely to attract tourists. As this part of the Atlas has only recently interested a few Moroccan or foreign geographers, this article is based on these few researches and especially on the numerous and punctual observations made, by the author, during the last two or three decades, until 1999.

\section{INDEX}

Mots-clés : Maroc, Anti-Atlas, tourisme culturel, patrimoine rural, désenclavement Keywords: Morocco, Anti-Atlas, cultural tourism, rural heritage, opening up

\section{AUTEUR}

\section{ANDRÉ HUMBERT}

Université de Lorraine, LOTERR, Nancy, 54000, France, tapisvolant4@gmail.com 